\title{
When Chronic Isn't Chronic: The Moderating Role of Active Self-Aspects
}

\author{
Christina M. Brown \\ Allen R. McConnell \\ Miami University
}

The current work considered how self-concept organization moderates the consequences of chronic attributes, which are widely assumed to be always accessible and influential. In Study 1, the accessibility of participants' chronic attributes was assessed before and after activating a self-aspect that was either relevant or irrelevant to participants' chronic attributes. Results showed that chronic attributes were more accessible when they were relevant to a participant's active self-aspect than when they were irrelevant to it. In Study 2, participants read ambiguous behaviors performed by others, some of which could be interpreted in line with their own chronic attribute or an alternative attribute. Participants were more likely to interpret behaviors as consistent with their own chronic attributes, but only when a relevant selfaspect had been previously activated. These studies suggest that chronicity can be moderated by self-aspect activation, consistent with the perspective that the self consists of multiple, context-dependent self-aspects.

Keywords: self-concept; chronicity; construct accessibility; social perception; self-aspects

$\mathrm{T}$ he perspective that people have core traits critical to their view of the world has been widely embraced (e.g., Allport, 1955; Kelley, 1972; Kelly, 1955; Markus, 1977). Presumably, the frequent use of psychological constructs leads to their habitual employment (e.g., Bruner, 1957; Kelly, 1955), affecting perceptions of the self and of others (e.g., Markus, 1977; Markus, Smith, \& Moreland, 1985). More recently, work on chronicity has continued in this tradition (e.g., Bargh, Bond, Lombardi, \& Tota, 1986; Higgins, 1989). Specifically, it is argued that chronic constructs are "always on" and thus continuously filter perceptions and shape behaviors. Because knowledge that is so accessible would have a tremendous impact on everyday social actions, understanding factors that moderate its pervasiveness would be quite important. Accordingly, the current work considers how self-concept representation qualifies the scope of chronic constructs.

In general, it is assumed that knowledge varies in its level of activation in memory, with constructs that are relatively greater in accessibility being more influential in social perception (Bruner, 1957). There are two primary ways in which accessibility has been shown to increase. First, recent construct use increases its activation in memory (e.g., Higgins, Rholes, \& Jones, 1977; Srull \& Wyer, 1979), although such accessibility decays with the passage of time (Higgins, 1989). The second way of increasing accessibility is through repeated and frequent use of a construct over time, which heightens its accessibility even in the absence of recent activation (e.g., Bargh, Lombardi, \& Higgins, 1988; Higgins, King, \& Mavin, 1982). It is at this point (i.e., accessibility without recent use) that constructs are deemed chronic. Often, one's chronic constructs reflect long-standing use throughout one's lifetime. For instance, children whose parents repeatedly stress the value of honesty during childhood may, later in life, evaluate their friends, lovers, and coworkers first and foremost in terms of honesty. It is this feature of chronicity (i.e., influence even without recent activation) that underscores its import.

Although constructs are hypothesized to become chronic following repeated activation over time (Bargh, 1982), self-relevant information is particularly likely to

Authors' Note: This work was supported by NIMH Grant MH068279, NSF Grant BCS 0601148, and the Lewis Endowed Professorship. Please address all correspondence to Christina Brown, Department of Psychology, Miami University, Oxford, OH 45056; e-mail: browncm5@muohio.edu.

PSPB, Vol. 35 No. 1, January 2009 3-15

DOI: $10.1177 / 0146167208324675$

(C) 2009 by the Society for Personality and Social Psychology, Inc. 
become chronic because individuals frequently consider, discuss, and experience their own traits, feelings, and behaviors. In fact, chronic constructs often take the form of self-schemata, which are chronic structures that organize information about oneself. Markus (1977) proposed that some traits (what she termed schematic traits) are so defining to a person that they become associated with the self-concept and thus play an important role in shaping information processing. These schematic traits are identified when people report that particular traits are especially important to them. For example, Markus found that participants who were schematic for independence or dependence, in comparison to those not schematic for those concepts, were faster to judge whether they possessed those traits on a speeded self-judgment task where they indicated, as quickly as possible, whether each trait was self-descriptive by pressing either a "me" or "not me" response key. The latency with which participants could render the judgment represented an index of how strongly the trait was associated with their self-concept. Those who were schematic for the traits had faster response latencies for self-judgments on those traits, indicating that traits identified as self-descriptive were accessible and strongly associated with the self-concept.

Overall, chronic constructs influence attention, interpretation, memory, and behavior (for an overview, see Higgins, 1989). For instance, Bargh (1982) found that information related to an individual's self-schemata is processed especially efficiently. In this study, participants who were either schematic or not for the trait "independence" performed a listening task in which they shadowed (i.e., spoke out loud) the words being presented, some of which were related to independence. In addition, participants were given a secondary task in which they were to monitor a light and press a button as quickly as possible once it was illuminated (it was lit at randomly determined intervals while they performed the shadowing task). This secondary task provided a measure of spare processing capacity in that participants could respond more quickly to the light probe if they had greater unused processing capacity available. Bargh found that when participants were shadowing independencerelated words, schematic participants were faster to respond to the probe than participants who were not schematic. In other words, constructs related to independence required less attentional resources by participants who were schematic for it, reflecting the relatively greater accessibility of chronic information.

In another study, Higgins et al. (1982) demonstrated that chronic constructs influence memory for constructrelevant information. For this study, chronic attributes were identified by having participants list up to 10 traits for each of the following categories: people they liked, people they disliked, people they sought out, people they avoided, and people they frequently encountered. The first trait listed (or the first unique trait listed if the first trait appeared in more than one category) from each category was considered a chronic trait. Then, 1 week later in an apparently unrelated experiment, participants read an essay containing a number of behaviors performed by a target individual. Half of the behaviors were descriptive of a trait that was chronic to them and the other half were descriptive of a trait chronic to another participant but not to themselves, producing a yoking procedure. Later, they were asked to reproduce the essay they had read about the target individual. Participants were more likely to remember behaviors describing a trait that was chronic to them than behaviors describing another participant's chronic trait (i.e., their yoked counterpart), suggesting a memorial advantage for chronic information.

Finally, the chronic accessibility of attributes can affect the interpretation of others' behaviors as well. Temporarily accessible constructs have long been known to guide the interpretation of ambiguous information, as shown with the classic "Donald paradigm" (Srull \& Wyer, 1979). In this work, participants read about a man named Donald whose ambiguous behaviors are subject to multiple interpretations. For instance, his behaviors could be descriptively hostile but ambiguous to the extent that they are caused by situational or dispositional factors. In the Bargh et al. (1986) study, participants interpreted Donald's behaviors as more dispositionally hostile when hostility was chronic for them.

In sum, there is considerable evidence that chronic information enjoys greater activation in memory, making it influential in social perception. Although it is presumed that such information is always accessible, there are reasons to suspect that its influence may be more limited. For example, concepts become schematic when they are associated with the self-concept and are highly accessible (Bargh, 1982). Yet, what does "associated with the selfconcept" mean? Indeed, there has been a growing recognition that the self-concept is multifaceted and context specific rather than a single, global entity (Baumeister, 1998; Linville \& Carlston, 1994; McConnell \& Strain, 2007; Mischel \& Morf, 2003; Schleicher \& McConnell, 2005). Therefore, it is possible that concepts may be highly accessible for some, but not all, facets of the self. If true, then chronicity-related outcomes (e.g., biased interpretations of the self and others) may not be revealed when context activates a subset of self-knowledge that is not associated with the "chronic" attributes. The current work examined this prediction in two studies.

Our thinking was guided by the multiple self-aspects framework (MSF; McConnell, 2007), which proposes that the self-concept is a comprised of a collection of multiple, context-dependent self-aspects. Self-aspects 
reflect idiosyncratic organizing contexts, such as roles (e.g., student), group identities (e.g., being Jewish), social relationships (e.g., with my spouse), affective states (e.g., when I'm moody), and behavioral situations (e.g., meeting new people), just to name a few. In general, selfaspects are broad organizing concepts, capturing roles (e.g., Roberts \& Donahue, 1994), goals (e.g., Higgins, 1997), private and public selves (e.g., Triandis, 1989), and relational and collective identities (e.g., Brewer \& Gardner, 1996). Each self-aspect in turn is associated with attributes, which can include traits (e.g., honest), behaviors (e.g., philanthropy), physical characteristics (e.g., attractive), and affective responses (e.g., happy), among others. Whereas self-aspects reflect organizing contexts that are extrapersonal (e.g., When I am, Where I am), attributes are the features exhibited by the person in those contexts that are intrapersonal (e.g., What I am, How I am). Thus, from the perspective of the MSF, the self can be viewed as a collection of self-aspects, each of which is associated with attributes, forming a broad, intricate associative network of self-knowledge.

There is evidence supporting this conceptualization of the self-concept. For example, McConnell, Rydell, and Brown (2008) had participants complete a self-concept description task that asked people to list their self-aspects and nominate the attributes associated with each from a list of 40 trait terms (Linville, 1985; McConnell et al., 2005; Showers, 1992). Several weeks later, they returned to the laboratory for what appeared to be an unrelated, threepart study. In the first and third experimental blocks, participants completed identical lexical decision tasks where the target words were the same 40 attributes provided to participants during the self-concept description task completed earlier in the semester. In between these lexical decision tasks, participants wrote for 5 minutes about one of their self-aspects that they listed in the self-concept description task. For example, a particular participant may have written about their typical day as a student (if they had reported having a student self-aspect). The writing exercise was designed to activate a particular self-aspect, which should increase the accessibility of only those attributes associated with it. Indeed, that is exactly what was found: Participants were faster (following the priming) with lexical judgments about attributes associated with the activated self-aspect (i.e., the one they wrote about) and not about attributes associated with other self-aspects. Thus, context (in this case, instantiated by the writing exercise) activated a self-aspect, which in turn increased the accessibility of attributes only associated with it.

One issue not addressed in the McConnell et al. (2008) study is whether chronic attributes are relatively accessible all of the time (the classic assumption of research on chronicity) or only when a relevant self-aspect is activated in memory (the MSF-derived prediction). Although the data seem consistent with the latter possibility (i.e., only attributes associated with the primed self-aspect showed an accessibility advantage), the authors did not assess the chronicity of attributes. Thus, it is unclear how accessible chronic attributes are when unrelated self-aspects are activated in memory. The current work explored this question.

Based on the MSF, we anticipated that the accessibility of attributes identified as chronic would be considerable when the currently active self-aspect is associated with these attributes (replicating past findings) but not when the currently active self-aspect is not associated with these attributes (a novel outcome). In contrast, the classic perspective would predict that chronic attributes would be highly accessible regardless of self-aspect activation. We examined these two hypotheses (i.e., classic vs. MSF-derived) in two studies for judgments about the self (Study 1) and for interpretation of others' ambiguous behaviors (Study 2).

\section{STUDY 1}

The current study evaluated the two predictions regarding judgments for the self, using a two-session design. In Session 1, we identified attributes that were chronic to participants using reaction time and self-report measures. Traits that participants responded to quickly on a self-judgment computer task and that were described as important in a self-report questionnaire were classified as chronic attributes (Markus, 1977). Participants also completed a self-concept description task, providing a description of their self-aspects and attributes associated with each self-aspect (McConnell et al., 2005).

In Session 2 (conducted at least 1 week later), attribute accessibility was measured using the same self-judgment task from Session 1. To the extent that response latencies for the attributes were relatively faster during Session 2 in comparison to Session 1, it would suggest that those attributes were relatively more accessible. To evaluate the predictions, we manipulated self-aspect activation prior to the Session 2 self-judgment task. Specifically, each participant was assigned to either a relevant self-aspect or irrelevant self-aspect condition. In the relevant self-aspect condition, participants wrote about a self-aspect that (based on their self-concept description responses) included a chronic attribute. Conversely, participants in the irrelevant self-aspect condition wrote about a selfaspect that was not associated with any of their chronic attributes. Based on the MSF, we expected relatively greater accessibility for the chronic attributes (as indicated by relatively faster self-judgments at Session 2 in comparison to Session 1), but only for those participants in the relevant self-aspect condition. On the other hand, 
we did not expect to see this accessibility advantage at Session 2 for participants in the irrelevant self-aspect condition, revealing the context-dependent nature of chronicity for the self. However, if the classic perspective of chronicity is correct, we would expect to see heightened accessibility of chronic attributes regardless of which self-aspects were activated.

\section{METHOD}

\section{Participants}

A sample of 48 undergraduate students at Miami University participated in return for course credit. Two additional participants' data were discarded because their responses indicated that they did not follow the instructions provided (i.e., many self-judgments were made in under 300 milliseconds, suggesting indiscriminant responding).

\section{Measures}

Assessing chronicity. Two tasks were used to identify participants' chronic attributes, one based on latency of self-judgments (e.g., Shah \& Higgins, 2001) and one based on ratings of trait importance (e.g., Markus, 1977). First, a me/not me (MNM) task presented each participant with a number of adjectives on a computer monitor one at a time. For each item, participants were asked to judge as quickly as possible whether the adjective described them by pressing one of two keys on the keyboard, labeled me and not me. The attributes, developed by Showers (1992), were 20 positive and 20 negative traits commonly used by undergraduates to describe themselves. The attributes were presented in six blocks of 40, with each attribute appearing once in each block. The order of attributes within each block was randomly determined. Each attribute remained on the screen until the participant indicated whether or not the attribute was self-descriptive. After the participant responded, a 1,000-millisecond blank screen was displayed before the next trial was presented. Participants were instructed to keep one finger on top of each key to facilitate responding. All trials with response latencies less than 300 milliseconds and greater than 3,000 milliseconds were removed from analyses $(<1 \%$ of all trials). After removing these trials, the participant's mean response latency across the six trials was calculated for each attribute.

The second task used to identify chronic attributes provided participants with the same list of 40 attributes used in the MNM task on a sheet of paper. In this trait importance task, participants rank-ordered the 5 attributes most important to themselves and the 5 attributes most important in other people. Specifically, for the attributes most important to themselves, they were told:

From the list on the right, please pick the 5 attributes you consider most important to your sense of who you are. It does not matter if the attribute is descriptive of you; in other words, it can be important to you because you don't have that attribute. Try to pick attributes that you think about frequently in your daily life when thinking about yourself, who you are, and who you are not. Order them from 1 to 5,1 being the most important and each successive number being less important.

For the attributes most important in other people, participants were told:

From the list on the right, please pick the 5 attributes you consider most important in other people; that is, choose the attributes that you look for in a friend. The attributes can be characteristic of what you seek, as well as characteristic of what you avoid. Try to pick attributes that you think about frequently in your daily life when thinking about other people, whether they are a good person, and whether you want to be their friend. Order them from 1 to 5,1 being the most important and each successive number being less important.

For an attribute to be considered chronic, the participant's mean response latency for it had to be among the 5 fastest response latencies (out of the 40 traits) and the participant had to select it as among the 5 most important in either how they saw themselves or how they saw other people. By requiring that an attribute be both highly accessible (based on response latencies) and selected as important, this provided a converging measure of chronicity for the participant. ${ }^{1}$ The mean number of chronic attributes for participants was $1.92(S D=0.90)$.

Self-concept description task. To assess self-concept representation, participants completed a computerized self-concept description task (McConnell et al., 2005; Renaud \& McConnell, 2002). Specifically, they were presented with the same list of 40 attributes used in the aforementioned tasks and asked to place them into groups that represent meaningful aspects of their lives (instructions were modeled after Linville, 1985). For each self-aspect (i.e., group they created), they provided a descriptive label. For example, participants may have placed intelligent, organized, and tense in one group and labeled it as their student self-aspect. It was emphasized that they did not have to use all of the attributes provided and that they could reuse the same attribute in multiple self-aspects. They were instructed to continue generating new self-aspects until they felt they had 
represented all of the important aspects of their lives. Furthermore, they were told that if they felt they were straining to generate more self-aspects, they should stop.

Self-aspect selection. We identified self-aspects that were relevant and irrelevant to participants' chronic attributes. Specifically, self-aspects were considered relevant if they contained at least one chronic attribute (as identified earlier), whereas self-aspects were considered irrelevant if they did not contain any chronic attributes. For participants with multiple chronic attributes, the relevant self-aspect selected was always the one that contained the most chronic attributes. In cases where the relevant self-aspect did not contain all of the participants' chronic attributes $(N=8 ; 16 \%$ of participants), only the chronic attributes included in that self-aspect were analyzed (i.e., the response latencies for chronic attributes not contained in the relevant self-aspect were excluded from analyses because those chronic attributes would not be relevant to the self-aspect under examination).

Measure of attribute accessibility. To assess changes in attribute accessibility during Session 2, participants completed the MNM task for a second time. To reduce participant fatigue, each of the 40 attributes was presented in three blocks (instead of six, as in Session 1), for a total of 120 trials. As with the data from the first MNM task, all trials with a response latency less than 300 milliseconds or greater than 3,000 milliseconds were removed $(<1 \%$ of all trials). Participants' mean response latencies during Session 1 were compared to those at Session 2 to assess changes in the accessibility of each attribute.

\section{Procedure}

Participants completed the experiment individually in small private rooms. At Session 1, they completed the MNM task, the trait importance task, and the selfconcept description task in a counterbalanced order. Later in the semester, participants were contacted by e-mail and invited to participate in a study (Session 2) that was portrayed as unrelated to Session 1.

At Session 2, participants wrote for 10 minutes about a particular self-aspect, which was preselected to be either relevant or irrelevant to the participant's chronic attributes (as described earlier). This activity was designed to activate the self-aspect in memory. For example, a participant who possessed a with family and friends self-aspect was told, "Please spend the next 10 minutes writing about how you feel when you're with your family and friends. Do not write about your family and friends themselves, but focus on how you feel, think, and behave when you are with your family and friends." Because the self-aspect written about (relevant or irrelevant to chronic attributes,

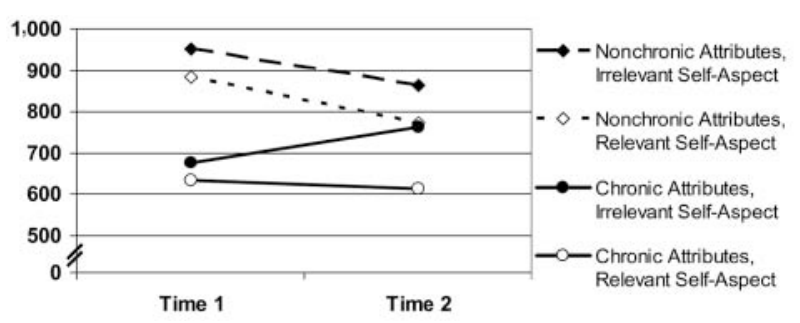

Figure 1 Accessibility of nonchronic and chronic attributes as a function of self-aspect activation and session (Time $1 \mathrm{vs}$. Time 2) in Study 1.

randomly assigned between participants) was idiosyncratic in nature (based on the labels they provided during the self-concept description task), the writing instructions were individually tailored for each participant.

Participants were instructed to write for 10 minutes, after which they completed the Session 2 MNM task. Finally, participants were debriefed and thanked for their participation.

\section{RESULTS}

\section{Attribute Accessibility}

To examine changes in accessibility of chronic and nonchronic attributes from Session 1 (before self-aspect activation) to Session 2 (following self-aspect activation), the mean latencies for chronic and nonchronic attributes on the MNM tasks were submitted to a 2 (active selfaspect: relevant vs. irrelevant) $\times 2$ (attribute type: chronic vs. nonchronic) $\times 2$ (session: Session 1 vs. Session 2) mixed-design ANOVA, with the latter two factors withinsubjects. As expected, the three-way interaction was significant, $F(1,46)=3.96, p=.05$ (see Figure 1). To better understand this effect, 2 (attribute type) $\times 2$ (session) repeated measures ANOVAs were conducted for the relevant and irrelevant self-aspect conditions separately.

For participants whose active self-aspect was irrelevant to their chronic attributes, the interaction between attribute type and session was significant, $F(1,20)=$ $18.23, p<.001$. These participants were faster to respond to nonchronic attributes at Session $2(M=$ $862.68, S D=184.04)$ than at Session $1(M=952.29$, $S D=181.16), F(1,20)=25.91, p<.001$. In contrast, responses to chronic attributes were slower at Session 2 $(M=762.23, S D=285.31)$ than at Session $1(M=$ $676.55, S D=147.30), F(1,20)=5.70, p<.05$. Thus, these participants revealed a general regression to the mean effect (i.e., faster Session 2 responses for attributes that were relatively slow at Session 1 and slower Session 
2 responses for attributes that were relatively fast at Session 1).

In the relevant self-aspect condition, there was also an interaction between session and attribute type, $F(1,26)=$ $19.68, p<.001$, but its form was different. Similar to the data for the irrelevant self-aspect condition, participants' responses to nonchronic attributes were faster at Session 2 $(M=772.63, S D=124.65)$ compared to Session 1 $(M=883.59, S D=150.12), F(1,26)=13.63, p<.01$. However, unlike the irrelevant self-aspect condition data, response latencies for chronic attributes did not change from Session $1(M=634.22, S D=94.88)$ to Session 2 $(M=613.33, S D=98.20), F(1,26)=.81$, ns. In other words, the activation of a self-aspect relevant to the chronic attributes offset the general regressive trend (i.e., slower Session 2 judgments in comparison to Session 1 judgments), indicating that the chronic attributes revealed relatively strong accessibility at Session 2, but only when a relevant self-aspect was activated in memory.

\section{DISCUSSION}

Overall, participants showed faster responses at Session 2 than at Session 1 for nonchronic attributes, consistent with practice and general regression to the mean effects based on the selection of chronic (identified by especially fast Session 1 responses) and nonchronic (identified by relatively slower Session 1 responses) attributes. Similarly, response latencies for the chronic attributes were slower in Session 2 than in Session 1, but only when an irrelevant self-aspect was activated in memory. In contrast, when a relevant self-aspect was active, response latencies for chronic attributes were unchanged. In other words, activating a self-aspect associated with chronic attributes maintained the strong accessibility of chronic attributes, whereas activating a self-aspect not associated with chronic attributes led to relatively slower judgments at Session 2. Overall, this demonstrates that chronic attributes reveal relatively heightened accessibility only when relevant self-aspects are activated in memory.

Thus, the current study indicates that the accessibility of chronic attributes is qualified by the currently active self-aspect. When a self-aspect that is unrelated to one's chronic attributes is activated, the accessibility advantage revealed by chronic attributes is relatively diminished. This indicates that the accessibility of chronic attributes can be qualified by which self-aspect is activated, which is more consistent with MSF-derived predictions than with predictions one would anticipate from a classical perspective on chronicity.

Although the current findings provide an intriguing demonstration of how chronicity is self-aspect dependent, some limitations exist. First, it would be desirable to demonstrate how self-aspect activation qualifies the consequences of chronic attributes where the outcome is less likely to be subject to practice and regression effects. Second, in addition to exploring the implications of chronicity for the self (Study 1), chronic attributes should also have an impact on perceptions of others (e.g., Bargh et al., 1986; Markus et al., 1985). Thus, we conducted a second study where the dependent measure of interest was less subject to restricted range effects and practice effects. Moreover, we examined the implications of chronic attributes for judgments of others' behaviors.

\section{STUDY 2}

The first study demonstrated that the accessibility of chronic attributes for judgments of the self is moderated by the currently active self-aspect. However, another important implication of chronic constructs is that they can influence perceptions of others (e.g., Bargh et al., 1986). Thus, in the current study we predicted that the influence of chronic constructs on perceptions of others would be diminished when one's currently active self-aspect is not associated with chronic attributes. To test this hypothesis, we first identified attributes that were chronic for each participant. Next, they returned to the laboratory for a second session where they read others' ambiguous behaviors that could potentially be construed as consistent with their chronic attributes. Prior to performing this ambiguous behavior judgment task, a manipulation either activated a relevant self-aspect or an irrelevant self-aspect. Finally, the current study used a different method for identifying chronic attributes (Higgins et al., 1982) that was not susceptible to regression effects and restricted range effects (cf. Study 1).

\section{METHOD}

\section{Participants}

A sample of 40 undergraduate students at Miami University participated in return for $\$ 10$ or for course credit. They participated in a two-session study, with a least 1 week between sessions.

\section{Measures}

Measure of chronicity. Chronic attributes were identified using Higgins et al.'s (1982) measure of chronicity. Specifically, participants were instructed to list up to 10 traits that belong in each of the following categories: people they like, people they dislike, people they seek out, people they avoid, and people they frequently 
encounter. Chronic attributes were those identified as the first attribute listed in each of the five categories. If the same attribute was listed first in more than one category, the next unique attribute listed in the category was used. As a result, all participants had five distinct chronic attributes in the current study.

Interpretation of ambiguous behaviors. Ambiguous behaviors were constructed based on participants' chronic attributes. For each chronic attribute, experimenters constructed a sentence that described another individual's behavior that could be interpreted as reflecting a chronic attribute or another attribute. Each participant read 10 sentences total. For example, "Jeff never says no when his friends ask for his help" could be interpreted as giving or submissive. In this case, giving would be the chronic attribute and submissive would be the alternative.

To ensure that chronic and alternative attributes did not vary in some unintended fashion, yoking between participants was used. For example, it may be that attributes reported as chronic vary in systematic ways (e.g., valence, frequency of occurrence, behavioral diagnosticity) from other attributes, and thus participants may be predisposed to interpret ambiguous behaviors as indicating chronic attributes. Thus, to ensure that perceptions of chronic attributes reflect their accessibility and not some unintended confound, participants read five additional sentences that could be interpreted in line with another person's chronic attribute or an alternative. If participants are more likely to interpret a behavior as a chronic attribute, regardless of whether it is their own or someone else's, then it would suggest that an extraneous quality of chronic attributes, and not their accessibility, made them preferable to the alternative attributes. However, if participants are only more likely to perceive behaviors as reflecting their own chronic attributes and not someone else's, then this more clearly indicates that the results are due to differences in construct accessibility instead of qualities of chronic attributes in general.

To achieve this end, 5 of the behavioral statements that each participant read could be interpreted as their ownchronic attribute or an alternative. The other 5 could be interpreted as someone else's chronic attribute (otherchronic) or an alternative. Specifically, participants were paired such that their own-chronic attributes that anchored one end of the rating scale for each ambiguous behavior served as the other-chronic attributes for their yoked partner (and accordingly, the participant's otherchronic attributes were their yoked partner's own-chronic attributes). The only criterion for yoking participants was that the 2 participants being yoked did not share any chronic attributes. Thus, each participant was asked to interpret 10 ambiguous behaviors, 5 of which could be interpreted as their own-chronic attribute or an alternative attribute (interpretable as own-chronic), and the other 5 of which could be interpreted as someone else's chronic attribute or an alternative (interpretable as other-chronic).

In all, 34 participants were yoked in reciprocal pairs. For these participants, the 5 ambiguous behaviors that were interpretable as other-chronic came from a participant who received their 5 interpretable as own-chronic behaviors in return (which then became the latter participant's interpretable as other-chronic behaviors). Also, 6 additional participants signed up for experimental sessions at a time when there were no participants who satisfied the yoking criteria. These participants were each yoked to a participant from a pool of more than 200 students who completed Session 1 but did not return for Session 2 (omitting these 6 participants from the analyses did not change the pattern of results). This process ensured that all participants read 5 ambiguous behaviors that could be interpreted as their own chronic attributes and another 5 that could be interpreted as someone else's chronic attributes.

After reading each ambiguous behavior, participants indicated on an 8-point scale the extent to which that behavior was 1 (descriptive of a chronic attribute, either own-chronic or other-chronic) or 8 (descriptive of another attribute unrelated to anyone's chronic attribute). For example, a female participant for whom outgoing was chronic (or for whom it was chronic to her yoked partner) would read, "Andrea begins conversations with everyone she meets." She would be asked to evaluate the implication of the behavior on a scale from 1 (outgoing) to 8 (intrusive). Participants always read behaviors performed by an actor whose gender was the same as their own. The appendix presents sample ambiguous behaviors and possible attribute interpretations. The order of the 10 behaviors was randomized so behaviors implying chronicity for the participant and for the yoked participant were interspersed.

Self-aspect selection. The method for selecting relevant and irrelevant self-aspects was the same as Study 1 with one modification to strengthen the relevancy manipulation. Specifically, because the current study (unlike the previous study) ensured that every participant had five chronic attributes, relevant self-aspects in Study 2 were those associated with at least two chronic attributes. As in Study 1, irrelevant self-aspects had no chronic attributes associated with them.

Because the measure of chronic attributes was openended, some of the attributes that participants generated were not among the 40 provided to them on the self-concept description task. In cases where a chronic attribute generated by the participant was a synonym for one of the 40 used in the self-concept description 
task, it was considered an instance of that attribute. For example, a participant who listed smart as a chronic attribute was considered to endorse its synonym intelligent, which was among the 40 attributes provided during the self-concept description task.

Postexperiment questionnaire. After rating the ambiguous behaviors, participants completed a questionnaire to examine self-aspect importance and to assess the relevancy of their chronic attributes to the self-aspect they had activated during the writing task. Specifically, participants were first asked to consider the self-aspect they wrote about and to indicate on scale ranging from 1 (not at all important) to 8 (very important) how important the self-aspect is to them. This question was used to ensure that the manipulation of self-aspect relevancy did not produce an unintended confound with self-aspect importance. In other words, it is possible that self-aspects relevant to one's chronic attributes are also more important, and thus any observed differences could reflect differential importance instead of relevance. Next, participants considered each of their five chronic attributes and indicated on a scale ranging from 1 (not at all relevant) to 8 (very relevant) how relevant each attribute was to that particular self-aspect. This served as a manipulation check to ensure that those who activated a relevant (vs. irrelevant) self-aspect during the writing exercise viewed the chronic attributes as relatively more relevant to it.

\section{Procedure}

At Session 1, participants completed the self-concept description task used in Study 1 as well as the measure of chronicity (order was counterbalanced). At least 1 week later, they were invited by e-mail to participate in a study in which they would write an essay about themselves and read about behaviors of other people. As in Study 1, Session 2 appeared to be unrelated to Session 1 .

During Session 2, participants began by writing an essay about either a relevant or irrelevant self-aspect for $10 \mathrm{~min}$ utes, as in Study 1. Next, participants completed the measure that assessed their interpretation of others' ambiguous behaviors. Afterward, they completed the postexperiment questionnaire to assess the importance of the activated selfaspect (i.e., the one they wrote about) and the relevancy of their chronic attributes for that self-aspect. Finally, all participants were debriefed and thanked.

\section{RESULTS}

\section{Manipulation Checks}

To ensure that participants' five chronic attributes were relevant or irrelevant to their active self-aspects as intended, the mean relevancy ratings for their chronic attributes was calculated. As expected, chronic attributes were judged to be significantly more relevant to the active self-aspect in the relevant condition $(M=$ $6.90, S D=.85)$ than in the irrelevant condition $(M=$ $4.16, S D=1.25), F(1,25)=43.88, p<.001$, indicating that the manipulation of relevance was effective.

Also, the activated self-aspect did not differ reliably in importance for those assigned to the relevant $(M=$ $7.40, S D=.70)$ and irrelevant $(M=6.75, S D=1.29)$ conditions, $F(1,20)=2.03$, ns, demonstrating that participants' active self-aspects were not perceived as more important when they were associated with chronic attributes than when they were not.

\section{Interpretation of Others' Ambiguous Behaviors}

The primary question of interest in Study 2 was whether participants would interpret others' ambiguous behaviors in line with their own chronic constructs, especially when a relevant self-aspect was activated in memory by the writing exercise. To address this question, participants' interpretations of the ambiguous behaviors (on the 8-point scale) were coded such that greater numbers indicated the behavior was interpreted as more in line with a chronic attribute (either their own or a yoked other's).

It is important to note that although participants rated ambiguous behaviors that applied to all five of their chronic attributes, not all five attributes were necessarily related to participants' relevant self-aspect. Selfaspects were considered relevant if they were associated with at least two chronic attributes, and for some participants these were the only chronic attributes associated with that self-aspect. In other words, a relevant self-aspect could have been relevant to only a subset of the participant's chronic attributes. Thus, ratings of own-chronic attributes only included chronic attributes reported as relevant for the particular self-aspect activated in the essay-writing task. Accordingly, the relative use of chronic attributes was analyzed using a 2 (selfaspect activation: relevant, irrelevant) $\times 2$ (type of chronic attribute: own-chronic, other-chronic) mixeddesign ANOVA, with the latter factor within-subjects. As Figure 2 reveals, the interaction was significant, $F(1,25)=5.24, p<.05$. When participants' active selfaspects were relevant to their chronic attributes, they were significantly more likely to interpret ambiguous behaviors as more consistent with their own chronic attributes instead of alternative attributes $(M=5.49$, $S D=1.05)$ than they were to interpret ambiguous behaviors as in line with someone else's chronic attributes instead of alternative attributes $(M=4.37$, $S D=.80), F(1,25)=12.90, p<.002$. However, when 


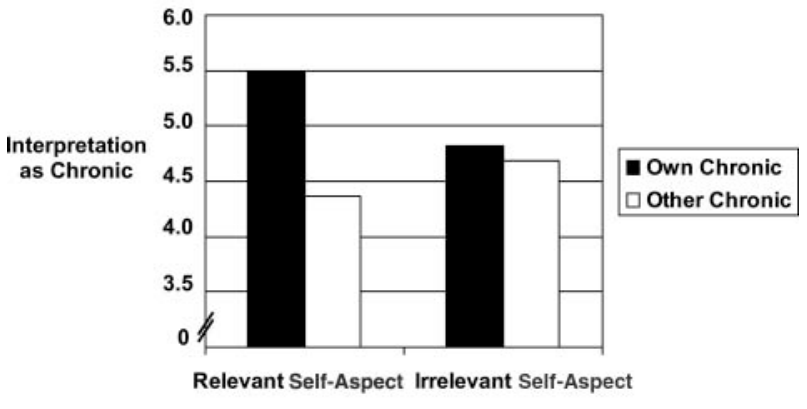

Figure 2 Mean rating of the extent to which ambiguous behaviors were viewed as relatively more chronic (own vs. other) as a function of self-aspect activation in Study 2.

participants' active self-aspects were irrelevant to their chronic attributes, they were no more likely to interpret ambiguous behaviors as relatively consistent with their own chronic attributes $(M=4.81, S D=.69)$ than as relatively more in line with someone else's chronic attributes $(M=4.69, S D=.54), F<1$.

\section{DISCUSSION}

The findings of Study 2 indicated that participants only used their chronic attributes to interpret others' ambiguous behaviors when their active self-aspect was associated with their chronic attributes. However, when their active self-aspect was irrelevant to their chronic attributes, participants were no more likely to interpret ambiguous behaviors as consistent with their own chronic attributes than they were to interpret them as in line with another person's chronic attributes. These findings suggest that chronic attributes do influence the interpretation of others' ambiguous behaviors, but only when relevant self-aspects are activated in memory. In other words, the use of chronic attributes (in this case, in interpreting the acts of others) was qualified by which self-aspect was activated in memory.

\section{GENERAL DISCUSSION}

By the very nature of the term, constructs that are chronic should be highly accessible and influence social cognitive outcomes regardless of context. In contrast to this classical view of chronicity, the current work considered a prediction derived from the MSF, which posits that the influence of chronic attributes depends on self-aspect activation. Specifically, the current work revealed that chronic attributes did not shape selfjudgments (Study 1) or interpretations of others' behaviors
(Study 2) when irrelevant self-aspects were activated. On the other hand, the activation of relevant self-aspects did lead chronic attributes to assimilate judgments of the self and others. In other words, it seems that constructs are chronic within relevant self-aspects instead of across one's entire self-concept, reaffirming the importance of viewing the self as a collection of multiple, context-dependent selves (e.g., Linville \& Carlston, 1994; McConnell, 2007; McConnell \& Strain, 2007; Mischel \& Morf, 2003).

Although the interaction pattern observed in both studies was clearly supportive of the MSF-derived predictions, a potential concern with the current work should be noted. Borrowing from past research (e.g., Shah \& Higgins, 2001), response latencies were used in Study 1 to identify chronic attributes, but this approach encouraged regression to the mean effects in our dependent measures. Despite the fact that such an underlying tendency would make the predicted (and observed) interaction pattern more difficult to obtain, it was clearly a less-than-ideal approach to identifying chronic constructs. This limitation, however, was addressed by the methodology employed in Study 2. Indeed, the predicted interaction was once again observed in this study under conditions where regression effects were eliminated.

It is important to note a second alternative explanation for the observed interaction between attribute type and self-aspect relevance. Rather than regression to the mean being responsible for slower responses to chronic attributes when an irrelevant self-aspect is active, it may be that these chronic attributes are actively inhibited when irrelevant to the current self-aspect. Previous research has found that concepts are inhibited when they conflict with a current goal, stereotype, or identity (e.g., Bodenhausen \& Macrae, 1998; Förster \& Liberman, 2007; Hugenberg $\&$ Bodenhausen, 2004). To test for the possibility of inhibition, we calculated the mean change in response latencies from Time 1 to Time 2 for chronic attributes in the irrelevant self-aspect condition (Study 1) and found this difference score, $M=-85.68$, was significantly less than 0 , $t(20)=-2.39, p<.05$, consistent with inhibition. That is, not only were chronic attributes less accessible when irrelevant (vs. relevant) to the active self-aspect, but their accessibility may have also been actively inhibited. Such inhibition might be especially important for constructs that typically enjoy an activation advantage in most circumstances (see Bruner, 1957). Regardless of whether significant inhibition is revealed when irrelevant self-aspects are activated, the current findings clearly demonstrate that chronicity is dependent on active self-aspects. Indeed, future research should explore the conditions under which inhibition is most likely to occur (e.g., perhaps self-aspects must be in direct behavioral conflict with each other for inhibition to result). 


\section{Implications for the Self}

It is interesting to consider the current work with other studies revealing the moderating role of active self-aspects or identities on general psychological processes. For example, Shih, Pittinsky, and Ambady (1999) demonstrated that stereotype-related performance decrements depend on the individual's active self-aspect. Specifically, they found that Asian American women performed worse on math tests when their female identity was salient (i.e., stereotype threat resulting from beliefs about women's poor math skills) than when their Asian identity was salient. Similarly, Asian American women performed better on a verbal test (a positive stereotype associated with women) when their female identity was salient than when their Asian identity was salient (Shih, Pittinsky, \& Trahan, 2006). Thus, the currently active self-aspect or identity for these individuals moderated their susceptibility to stereotype threat and consequently their subsequent math or verbal performance.

Moreover, active self-aspects or identities have also been found to moderate the accessibility of related concepts. For instance, Hugenberg and Bodenhausen (2004) recruited participants who did or did not belong to a Greek organization (i.e., fraternity or sorority). In this study, some participants' Greek or non-Greek identity was primed, which was followed by a lexical decision task containing words related to Greek life (e.g., party, $k e g$ ) or words related to student life (e.g., study, read). Participants who were members of a Greek organization and had their Greek identity activated responded more quickly to the Greek-related words and more slowly to the student-related words compared to those whose Greek identity was not activated. That is, Greek-related constructs were more accessible but only when a related identity was active. Findings such as these demonstrate that the activation of a particular self-aspect can result in differential accessibility of knowledge associated with a related social identity, and the current study reveals that such outcomes even apply to knowledge assumed to always be highly accessible (i.e., chronic concepts).

More broadly, the current findings have important implications for research on the self in general. The results of these two studies are consistent with an increasing emphasis on the importance of multiple selves in studying self-relevant processes (e.g., Linville, 1985; Linville \& Carlston, 1994; Markus \& Nurius, 1986; McConnell et al., 2008; Shih et al., 1999; Showers, 1992). In particular, the MSF (McConnell, 2007) proposes that the self is best understood as being a collection of multiple, context-dependent self-aspects rather than as a unitary structure (see also, Markus \& Kitayama, 1991). This perspective on self-concept representation is supported by the current work demonstrating that chronic accessibility is dependent on active self-aspects, which is quite at odds with the view of a single self. This latter approach instead would conceive of the self as a stable and unitary concept with chronic constructs that never vary in their accessibility. The current findings in contrast support the position of multiple, context-dependent selves outlined by the MSF (McConnell, 2007; McConnell \& Strain, 2007).

The MSF perspective articulates a number of intriguing predictions in addition to the limits of chronicity. For instance, the experience of cognitive dissonance and subsequent justification efforts may depend on the importance of the dissonance-arousing behavior to the active self-aspect. Rather than viewing one's behavior or attitudes as "in conflict with the self" (which presupposes a unitary self), the MSF would predict that dissonance may or may not be experienced based on which self-aspects are activated in memory. Relatedly, self-affirmation (Steele, 1988) may successfully address dissonance through a variety of means, including increasing the positivity of the currently active (and challenged) self-aspect or by activating another positive self-aspect. Similarly, stereotyping and outgroup derogation may depend on whether an individual's active self-aspect is relevant to his or her group identity. In fact, the common ingroup identity model (Dovidio, Gaertner, \& Validzic, 1998) proposes that activating a superordinate ingroup identity reduces intergroup hostility, implying that prejudice and discrimination may depend on the relation between group identity and the individual's active self-aspect.

\section{Implications for Chronicity}

At the same time our findings underscore the importance of active self-aspects, they also reveal insights for the concept of chronicity. Chronically accessible concepts were originally viewed as concepts that are highly accessible and influential at all times (e.g., Bargh et al., 1988; Higgins, 1989). The current findings, however, challenge whether concepts can be highly accessible independent of contexts and their associated self-aspects.

We propose that certain concepts can be especially accessible when a particular self-aspect is activated. In other words, these concepts are "chronic" for a particular self-aspect. Does this mean that "chronic" attributes will only be applicable to one context? Not necessarily. For example, within the MSF, some attributes can be associated with multiple self-aspects, and it is quite possible that such attributes possess relatively greater accessibility (e.g., their importance is belied by their being relevant to multiple contexts, resulting in the appearance of constant accessibility), thus serving to direct perception and behavior to an especially strong degree. Moreover, 
there may be situations where context activates multiple self-aspects concurrently (e.g., a Jewish student enrolled in a class on Judaic studies may experience the simultaneous activation of student and Jewish self-aspects), and thus highly accessible attributes associated with either self-aspect may play an important role in guiding one's actions. ${ }^{2}$ In sum, our perspective rejects the existence of concepts that are chronically accessible regardless of current context (i.e., self-aspects), unless a critical attribute is associated with every self-aspect (certainly possible, but very unlikely). Thus, we view "chronic" as being highly accessible within a particular context.

If the self-concept is necessarily involved in all forms of chronicity, one might wonder why chronic accessibility is sometimes measured by asking participants to describe other people (e.g., Higgins et al.'s [1982] measure of chronicity; see also Study 2). We believe that the attributes people use to describe others are indeed selfrelevant, an association that is clearly supported by social perception research. Dunning (2003) provides a compelling review of research indicating that the self is always involved in social perception. Specifically, the self-concept is used as a basis for perceiving and evaluating other people. This does not mean that every chronic attribute used to describe other individuals is also self-descriptive. In fact, an attribute may often be used as a basis for judging others precisely because people believe it is not characteristic of themselves. For example, dishonesty can be a chronic attribute for those who see themselves as honest and detest dishonesty in others. Thus, while not self-descriptive, a chronic attribute can still be self-relevant. ${ }^{3}$ For these reasons, an attribute that is identified as chronic within a particular self-aspect should operate in the same way regardless of whether it is used to describe oneself or others.

\section{Conclusions}

In sum, the current work highlights the importance of considering self-concept structure in social perception. To the extent that self-relevant knowledge exists in an intricate network in memory, context should produce modulated activations of such networks, resulting in variability in what is accessible in memory and thus influential in guiding behavior and judgment at any given moment (see also, Mischel \& Morf, 2003; Schleicher \& McConnell, 2005; Smith, 2002). The current work demonstrates that such variability even exists for chronic constructs, which previously were assumed to influence interpretation and behavior regardless of context and circumstance. As a result, the current work reaffirms the importance of understanding how situations and context shape action even for constructs presumed to be always central and accessible to the individual.

\section{APPENDIX \\ SAMPLE OF 20 AMBIGUOUS BEHAVIORS AND ATTRIBUTE INTERPRETATIONS}

Honest versus rude: told her mother that she didn't like the gift she gave her.

Caring versus clingy: When her boyfriend was driving at night, called him three times to make sure he was okay.

Funny versus insensitive:

Snobby versus proud: likes to tease her friends about the clothes they wear.

told her friends about the new high-paying promotion her father got.

Friendly versus dependent: ___ tries to have lunch with her best friend almost every day of the week.

Successful versus lucky: ___ has never been turned down when applying for a job.

Pessimistic versus realistic: ___ didn't try out for a competitive soccer team because she figured she wouldn't get picked anyway.

Self-centered versus focused:___ couldn't help a friend with a problem because she needed to prepare for a job interview.

Cocky versus confident: ___ has no doubt that she will ace her history exam today.

Dishonest versus considerate: A guy was interested in dating

boyfriend so she wouldn't hurt his feelings.

Intelligent versus know-it-all: ___ raises her hand for every question in class.

Close-minded versus committed: After reading a new scientific article, ___ refused to believe the new theory because it was inconsistent

with her own research.

Bossy versus assertive: ___ always becomes the leader of class groups; she organizes the materials and decides how the tasks should be split among everyone.

Open-minded versus adventurous: is always willing to go to new restaurants and try new activities.

Classy versus materialistic: When it comes to restaurants, clothing, locations, etc., ___ will never settle for second best.

Ignorant versus biased: ___ did not look into the opinions of different political parties and voted for the party that her parents always

chose when she was growing up.

Laid back versus lazy: When the phone in ___ 's dorm room broke, she decided there was no reason to fix it.

Humble versus insecure: Whenever people compliment her, ___ gets red in the face and says "it's no big deal.”

Mean versus easily amused:

Thoughtless versus forgetful: laughed when a stranger slipped on the ice and fell unharmed. often borrows her roommate's stuff without asking first.

NOTE: The gender of the name and pronoun always matched the participant's gender. Female names: Andrea, Sarah, Jessica, Marie, Amanda, Stephanie, Jen, Tara, Christine, Julie; male names: Andrew, Sam, Jason, Matt, Anthony, Steven, Jeff, Tim, Christopher, John. 


\section{NOTES}

1. Because chronic attributes were identified in part based on especially fast response latencies during the Session 1 me/not me (MNM) task, the likelihood that regression to the mean effects would be observed during the Session 2 MNM task increases. Although the likelihood of regression to the mean may be considerable, the MSFderived prediction anticipates that relative differences in MNM latencies (Session 1 vs. Session 2) will be qualified by the type of self-aspect activated (i.e., relevant vs. irrelevant). In Study 2, we used other widely used methods for identifying chronic attributes that are not subject to potential regression to the mean effects.

2. On the other hand, there may be moments when no self-aspect is activated at all, such as conditions involving deindividuation or greatly reduced self-awareness. Although our current research cannot speak to the nature of chronic concepts when no self-aspects are active, we believe chronicity cannot exist in these situations because chronic concepts must be associated with the self (specifically, one or more self-aspects) in some way. Admittedly, our argument is speculative, and additional research is required to explore such possibilities.

3. Although we argue that chronic attributes that are descriptive of others have the same effects as self-descriptive chronic attributes, it is interesting to note that one's own attributes tend to be used when describing and evaluating others (e.g., Dunning, 2003; Sedikides \& Skowronski, 1993). Indeed, the fact that our Study 2 participants had self-aspects associated with chronic attributes that were identified using a person-description task indicates that chronic attributes are intimately tied to the self-concept.

\section{REFERENCES}

Allport, G. W. (1955). Becoming. New Haven, CT: Yale University Press. Bargh, J. A. (1982). Attention and automaticity in the processing of self-relevant information. Journal of Personality and Social Psychology, 43, 425-436.

Bargh, J. A., Bond, R. N., Lombardi, W. J., \& Tota, M. E. (1986). The additive nature of chronic and temporary sources of construct accessibility. Journal of Personality and Social Psychology, 50, 869-878.

Bargh, J. A., Lombardi, W. J., \& Higgins, E. T. (1988). Automaticity of chronically accessible constructs in Person $\times$ Situation effects on person perception: It's just a matter of time. Journal of Personality and Social Psychology, 55, 599-605.

Baumeister, R. F. (1998). The self. In D. T. Gilbert, S. T., Fiske, \& G. Lindzey (Eds.), Handbook of social psychology (4th ed., Vol. 1, pp. 680-740). New York: McGraw-Hill.

Bodenhausen, G. V., \& Macrae, C. N. (1998). Stereotype activation and inhibition. In R. S. Wyer (Ed.), Stereotype activation and inhibition: Advances in social cognition (Vol. 11, pp. 1-52). Mahwah, NJ: Lawrence Erlbaum.

Brewer, M. B., \& Gardner, W. (1996). Who is this "We"? Levels of collective identity and self representations. Journal of Personality and Social Psychology, 71, 83-93.

Bruner, J. S. (1957). On perceptual readiness. Psychological Review, 64, 123-152.

Dovidio, J. F., Gaertner, S. L., \& Validzic, A. (1998). Intergroup bias: Status, differentiation, and a common in-group identity. Journal of Personality and Social Psychology, 75, 109-120.

Dunning, D. (2003). The relation of self to social perception. In M. R. Leary \& J. P. Tangney (Eds.), Handbook of self and identity (pp. 421-441). New York: Guilford.

Förster, J., \& Liberman, N. (2007). Contrast effects in knowledge activation: The case of inhibition effects due to competing constructs or goal fulfillment. In D. A. Stapel \& J. Suls (Eds.), Assimilation and contrast in social psychology (pp. 269-287). New York: Psychology Press.

Higgins, E. T. (1989). Knowledge accessibility and activation: Subjectivity and suffering from unconscious sources. In J. S. Uleman \& J. A. Bargh (Eds.), Unintended thought (pp. 75-123). New York: Guilford.
Higgins, E. T. (1997). Beyond pleasure and pain. American Psychologist, $52,1280-1300$.

Higgins, E. T., King, G. A., \& Mavin, G. H. (1982). Individual construct accessibility and subjective impressions and recall. Journal of Personality and Social Psychology, 43, 35-47.

Higgins, E. T., Rholes, W. S., \& Jones, C. R. (1977). Category accessibility and impression formation. Journal of Experimental Social Psychology, 13, 141-154.

Hugenberg, K., \& Bodenhausen, G. V. (2004). Category membership moderates the inhibition of social identities. Journal of Experimental Social Psychology, 40, 233-238.

Kelley, H. H. (1972). Attribution in social interaction, In E. E. Jones, D. E. Kanouse, H. H. Kelley, R. E. Nisbett, S. Valins, \& B. Weiner (Eds.), Attribution: Perceiving the causes of behavior (pp. 1-26), Morristown, NJ: General Learning Press.

Kelly, G. A. (1955). The psychology of personal constructs (Vols. 1, 2). New York: Norton.

Linville, P. W. (1985). Self-complexity and affective extremity: Don't put all of your eggs in one cognitive basket. Social Cognition, 3, 94-120.

Linville, P. W., \& Carlston, D. E. (1994). Social cognition perspective on self. In P. G. Devine, D. L. Hamilton, \& T. M. Ostrom (Eds.), Social cognition: Contributions to classic issues in social psychology (pp. 143-193). New York/Berlin: Springer-Verlag.

Markus, H. (1977). Self-schemata and processing information about the self. Journal of Personality and Social Psychology, 35, 63-78.

Markus, H., \& Nurius, P. (1986). Possible selves. American Psychologist, 41, 954-969.

Markus, H., Smith, J., \& Moreland, R. L. (1985). Role of the selfconcept in the perception of others. Journal of Personality and Social Psychology, 49, 1494-1512.

Markus, H. R., \& Kitayama, S. (1991). Culture and the self: Implications for cognition, emotion, and motivation. Psychological Review, 98, 224-253.

McConnell, A. R. (2007). The multiple self-aspects framework: Selfconcept representation and its implications. Manuscript submitted for publication.

McConnell, A. R., Renaud, J. M., Dean, K. K., Green, S. P., Lamoreaux, M. J., Hall, C. E., et al. (2005). Whose self is it anyway? Self-aspect control moderates the relation between self-complexity and wellbeing. Journal of Experimental Social Psychology, 41, 1-18.

McConnell, A. R., Rydell, R. J., \& Brown, C. M. (2008). On the experience of self-relevant feedback: How self-concept organization influences affective responses and self-evaluations. Manuscript submitted for publication.

McConnell, A. R., \& Strain, L. M. (2007). Content and structure of the self. In C. Sedikides \& S. Spencer (Eds.), The self in social psychology (pp. 51-73). New York: Psychology Press.

Mischel, W., \& Morf, C. C. (2003). The self as a psycho-social dynamic processing system: A meta-perspective on a century of the self in psychology. In M. R. Leary \& J. P. Tangney (Eds.), Handbook of self and identity (pp. 15-43). New York: Guilford.

Renaud, J. M., \& McConnell, A. R. (2002). Organization of the selfconcept and the suppression of self-relevant thoughts. Journal of Experimental Social Psychology, 38, 79-86.

Roberts, B. W., \& Donahue, E. M. (1994). One personality, multiple selves: Integrating personality and social roles. Journal of Personality, 62, 199-218.

Schleicher, D. J., \& McConnell, A. R. (2005). The complexity of selfcomplexity: An associated systems theory approach. Social Cognition, 23, 387-416.

Sedikides, C., \& Skowronski, J. J. (1993). The self in impression formation: Trait centrality and social perception. Journal of Experimental Social Psychology, 29, 347-357.

Shah, J., \& Higgins, E. T. (2001). Regulatory concerns and appraisal efficiency: The general impact of promotion and prevention. Journal of Personality and Social Psychology, 80, 693-705.

Shih, M., Pittinsky, T. L., \& Ambady, N. (1999). Stereotype susceptibility: Identity salience and shifts in quantitative performance. Psychological Science, 10, 80-83.

Shih, M., Pittinsky, T. L., \& Trahan, A. (2006). Domain-specific effects of stereotypes on performance. Self and Identity, 5, 1-14. 
Showers, C. (1992). Compartmentalization of positive and negative self-knowledge: Keeping bad apples out of the bunch. Journal of Personality and Social Psychology, 62, 1036-1049.

Smith, E. R. (2002). Overlapping mental representations of self and group: Evidence and implications. In J. P. Forgas \& K. D. Williams (Eds.), The social self: Cognitive, interpersonal, and intergroup perspectives (pp. 21-35). New York: Psychology Press.

Srull, T. K., \& Wyer, S. (1979). The role of category accessibility in the interpretation of information about persons: Some determinants and implications. Journal of Personality and Social Psychology, 37, 1660-1672.
Steele, C. M. (1988). The psychology of self-affirmation: Sustaining the integrity of the self. In L. Berkowitz (Ed.), Advances in experimental social psychology (Vol. 21, pp. 261-302). San Diego, CA: Academic Press.

Triandis, H. C. (1989). The self and social behavior in differing cultural contexts. Psychological Review, 96, 506-520.

Received January 25, 2008

Revision accepted July 11, 2008 